July 2005

\title{
MULTIPLICATIVE BACKGROUND RISK *
}

\author{
Günter Franke, University of Konstanz, Germany \\ (Guenter.Franke@uni-konstanz.de) \\ Harris Schlesinger, University of Alabama, USA \\ (hschlesi@cba.ua.edu) \\ Richard C. Stapleton, University of Manchester, England \\ (richard.stapleton1@btinternet.com)
}

\begin{abstract}
$\underline{\text { Abstract }}$
Although there has been much attention in recent years on the effects of additive background risks, the same is not true for its multiplicative counterpart. We consider random wealth of the multiplicative form $\tilde{x} \tilde{y}$, where $\tilde{x}$ and $\tilde{y}$ are statistically independent random variables. We assume that $\tilde{x}$ is endogenous to the economic agent, but that $\tilde{y}$ is an exogenous and nontradable background risk, which represents a type of market incompleteness. Our main focus is on how the presence of the multiplicative background risk $\tilde{y}$ affects risk-taking behavior for decisions on the choice of $\tilde{x}$. We extend the results of Gollier and Pratt (1996) to characterize conditions on preferences that lead to more cautious behavior.
\end{abstract}

Keywords: affiliated utility function, background risk, diffidence theorem, multiplicative risks, multiplicative risk vulnerability, risk vulnerability

JEL Classification No.: D81

\footnotetext{
* The authors thank seminar participants at Duke University, the Wissenschaftszentrum-Berlin, the Universities of Alabama, Konstanz, Melbourne and Pennsylvania, as well as participants at the Risk Theory Seminar in Champaign, Illinois, the SIRIF Conference on Strategic Asset Allocation in Glasgow and the Meeting of the European Group of Risk \& Insurance Economists in Nottingham for helpful comments. We also are grateful to Axel Adam-Müller, Louis Eeckhoudt, Christian Gollier, Miles Kimball, Bob Nau, Luisa Tibiletti, Nicolas Treich and three referees for helpful comments.
} 


\section{Introduction}

Consider a risk-averse economic agent whose preferences can be represented within an expected-utility framework via the continuously differentiable von NeumannMorgenstern utility function of final wealth $u$. The agent must decide upon choice parameters for a random variable representing final wealth, $\tilde{x}$. For example, $\tilde{x}$ might represent wealth from an individual's portfolio of financial assets, or $\tilde{x}$ might represent random corporate profits based on management decisions within the firm.

Much attention in recent years has been given to how decisions on $\tilde{x}$ might be affected by the addition of an additive risk $\widetilde{\mathcal{E}}$, where $\widetilde{\mathcal{E}}$ and $\tilde{x}$ are statistically independent. Thus, final wealth or profits can be written as $\tilde{x}+\tilde{\varepsilon}$. The market is assumed to be incomplete in that $\widetilde{\varepsilon}$ is not insurable. For example, $\widetilde{\varepsilon}$ might represent future wage income subject to human-capital risks; or $\widetilde{\varepsilon}$ might represent an exogenous pension portfolio provided by one's employer. Although it is interesting to examine the interdependence between $\tilde{x}$ and $\widetilde{\varepsilon}$, the case of independence is of special interest and provides for many interesting observations. In order to focus on the risk effects, rather than wealth effects, it is often assumed that $E \widetilde{\varepsilon}=0$, where $E$ denotes the expectation operator. In such a case, $\widetilde{\varepsilon}$ is called a "zero-mean background risk." However, much of the literature focuses instead on the case where $E \tilde{\varepsilon} \leq 0$, in which case we call $\tilde{\varepsilon}$ an "undesirable background risk." Our purpose in the present paper is to examine the effects of introducing an independent "multiplicative background risk" into the individual's final wealth distribution. 
The modern literature on additive background risk stems from the papers of Kihlstrom, et al. (1981), Ross (1981) and Nachman (1982). ${ }^{1} \quad$ These papers focus on interpersonal behavior comparisons, mainly addressing the question: "If I am willing to pay more than you to rid myself of any fair lottery, would I still be willing to do so in the presence of an additive background risk?" Doherty and Schlesinger (1983) extended the analysis to intrapersonal models of decision making under uncertainty, focusing on differences in optimal behavior with vs. without a background risk. The literature underwent somewhat of a renaissance in the 1990's thanks to new theoretical tools provided by Pratt and Zeckhauser (1987) and Kimball (1990, 1993).

One canonical hypothesis concerning additive background risk is that the riskiness of $\widetilde{\varepsilon}$ leads to a more cautious behavior towards decisions on $\tilde{x}_{.}{ }^{2}$ For example, Guiso, et al. (1996) use Italian survey data to show that individuals with a riskier perception of their (exogenously managed) pension wealth react by investing relatively more in bonds in their personal accounts. However, this conclusion cannot be guaranteed theoretically unless particular restrictions on preferences are met. Eeckhoudt and Kimball (1992) first examined this direction of research. The strongest result to date is by Gollier and Pratt (1996), who characterize necessary and sufficient conditions for every undesirable additive background risk $(E \tilde{\varepsilon} \leq 0)$ to lead to more cautious behavior. They label this property "risk vulnerability." Since the conditions for risk vulnerability are rather complex, they also provide a few sufficient conditions for more cautious behavior that are much easier to verify.

Surprisingly, very little attention has been given to the case where the background risk is multiplicative. Exceptions are the papers by Nachman (1982), Pratt (1988) and Finkelshtain, et al. (1999), who all consider wealth to be a general function of two

\footnotetext{
${ }^{1}$ Nachman (1982) actually considers a more general structure than just an additive one, but still is considered a founding source for the additive case. Ross assumes zero correlation and "mean independence," rather than independence.

${ }^{2}$ By "more cautious behavior" we mean behavior that is consistent with the behavior of a more risk-averse individual.
} 
random variables, including multiplicative as a special case. The first two of these papers consider the interpersonal comparison of risk aversion both with and without the presence of an independent background risk. The Finkelshtain, et al. paper considers conditions on a bivariate utility function and on the two risks that maintain aversion to one risk, in the presence of the other (background) risk.

Our focus in this paper is on the intrapersonal comparison of behavior with and without an independent multiplicative background risk. Under what conditions on preferences will the presence of the multiplicative background risk compel the agent to behave more cautiously? Under what conditions will such a background risk lead the agent to behave in a less-cautious manner? Unlike the case for an additive background risk, it is not clear that more-cautious behavior should be expected when the risk is multiplicative. There are no empirical results that we are aware of relating to morecautious behavior for the multiplicative case. Our analysis shows that less-cautious behavior can follow under reasonable assumptions as well.

To this end, let $\tilde{y}$ be a random variable on a positive support that is statistically independent of $\tilde{x}$. Final wealth is given by the product $\tilde{x} \tilde{y}$. Examples of such multiplicative risks include the following:

1. Let $\tilde{x}$ be the pre-tax profits of a firm and let $\tilde{y}$ represent the firm's retention rate net of taxes, where tax rates are random due to tax-legislation uncertainty.

2. Let $\tilde{x}$ be portfolio wealth in period one, and let $\tilde{y}$ denote the return on a mandatory annuity account that rolls over the proceeds from $\tilde{x}$ in period two.

3. Let $\tilde{x}$ denote nominal wealth or profit and let $\tilde{y}$ denote an end-of-period price deflator. 
4. Let $\tilde{x}$ denote the random quantity of output for a farm commodity and let $\tilde{y}$ denote an exogenous random per-unit profit.

In order to isolate the risk effects of $\tilde{y}$, one can assume that $E \tilde{y}=1^{3}$ However, to maintain comparability with the additive-risk literature on "risk vulnerability," we also consider "an undesirable multiplicative background risk," with $E \tilde{y} \leq 1$.

Since $\tilde{x} \tilde{y}=\tilde{x}+\tilde{x}(\tilde{y}-1)$, the assumption that $E \tilde{y} \leq 1$, together with the independence of $\tilde{x}$ and $\tilde{y}$, guarantees that $\tilde{x} \tilde{y}$ is riskier than $\tilde{x}$ alone in the sense of second-order stochastic dominance. Defining $\tilde{\varepsilon} \equiv \tilde{x}(\tilde{y}-1)$, we see that $\tilde{x} \tilde{y}$ is a special case of additive risks, but that the risk $\tilde{\varepsilon}$ so defined is not independent of $\tilde{x}$, and thus not an "independent additive background risk" as defined above. ${ }^{4}$

The results for the multiplicative case do not simply mirror those of the additive case. To see this, consider a simple portfolio example with an allocative choice between risky stocks and risk-free bonds. An individual has an initial wealth of 100 and the riskfree rate is assumed to be $r_{f}=0.05$. The return on the stock portfolio is assumed to be $\log$-binomial with an expected return of $E \tilde{r}=0.11$ and a standard deviation of $\sigma=0.20$ (implying that, in a binomial model, stocks either return about $33 \%$ or lose about $10 \%$, each with an equally likely chance). Utility is assumed to belong to the HARA class with $u(x)=-\frac{1}{2}(x+a)^{-2}$, where $a$ is a constant chosen such that $x+a$ remains positive over relevant wealth levels. Preferences satisfy decreasing absolute risk aversion (DARA) for any choice of $a$, whereas relative risk aversion will be increasing [decreasing, constant] whenever $a$ is positive [negative, zero]. We examine two alternative sources of background risk: (1) an additive background risk, for which final wealth is either

\footnotetext{
${ }^{3}$ For instance, in our first example above we can let $\tilde{x}$ represent after-tax profits based on the expected tax rates and let $\tilde{y}$ represent an unexpected deviation from the expected after-tax retention rates

${ }^{4}$ The risk $\tilde{\varepsilon}$ so defined does satisfy Ross's condition of mean independence when $E \tilde{y}=1$. In this case, $\tilde{x} \tilde{y}$ is riskier than $\tilde{x}$ in the sense of Rothschild and Stiglitz (1970). However, $\tilde{\varepsilon}$ so defined is not exogenous if the risk $\tilde{x}$ is endogenous and any change in $\tilde{x}$ will alter the background risk as well.
} 
increased or decreased by 30 , each with probability one-half, or (2) a multiplicative background risk, for which wealth is either increased or decreased by 30 percent, each with a probability one-half. Optimal portfolio choices are illustrated in Table 1.

\section{TABLE 1: Bond Proportions: Multiplicative vs. Additive Background Risk}

(All utility is DARA within the HARA class, $u(x)=-\frac{1}{2}(x+a)^{-2}$, initial wealth $=100$ )

(Relative risk aversion is constant for $\mathrm{a}=0$, increasing for $\mathrm{a}=+25$ and decreasing for $\mathrm{a}=-25$ )

\begin{tabular}{l|l|c}
\hline Utility & Background Risk & Proportion in Bonds \\
\hline$a=0$ & None & $55 \%$ \\
& Additive & $66 \%$ \\
& Multiplicative & $55 \%$ \\
\hline \multirow{2}{*}{$a=+25$} & None & $45 \%$ \\
& Additive & $54 \%$ \\
& Multiplicative & $41 \%$ \\
& & \\
\hline \multirow{2}{*}{$a=-25$} & None & $66 \%$ \\
& Additive & $78 \%$ \\
& Multiplicative & $70 \%$ \\
\hline
\end{tabular}

Since, within the HARA class of preferences, an assumption of DARA also implies standard risk aversion (Kimball 1993), we know that bond proportions will always increase with an additive background risk. However, as the example shows, a multiplicative background risk might cause the bond proportion to shrink. When $a=25$, so that we have both DARA and increasing relative risk aversion - hardly considered unusual cases - we then have a lower proportion of wealth invested in the risk-free bond.

We show how each of the situations in the example above can be determined qualitatively (i.e. whether more or fewer bonds are purchased in the presence of a background risk) before calculating the optimal portfolios. The fact that the qualitative effects might be predetermined by the parameters of the model implies that care must be taken when modeling various economic and/or financial phenomena. For example, 
seemingly innocuous assumptions made about preferences might predispose a model to achieve particular results.

We begin in the next section with our model and then proceed to our main result, establishing necessary and sufficient conditions for an individual always to exhibit more cautious behavior in the presence of an independent multiplicative background risk. We compare our results to the additive case of "risk vulnerability" as defined by Gollier and Pratt (1996). Since the conditions we derive are rather technical, we also present some sufficient conditions that are quite easy to verify. We next introduce a device we call the "affiliated utility function," which we define as the composite of utility with the exponential function. Although the affiliated utility function is shown to have fairly restricted applicability, it does allow us to translate a few known results from the case of additive background risk to our model with multiplicative background risk. .

\section{The Model}

We wish to determine how a multiplicative background risk $\tilde{y}$ affects decision making on $\tilde{x}$. Both $\widetilde{x}$ and $\widetilde{y}$ are assumed to be strictly positive. Let $F$ and $G$ denote the (cumulative) distribution functions associated with $\tilde{x}$ and $\tilde{y}$ respectively. We also assume that the support of $G$ is bounded in the interval $[a, b]$. Since $\tilde{x}$ and $\tilde{y}$ are independent, we can write expected utility as the iterated integral

$$
E u(\tilde{x} \tilde{y})=\int_{0}^{\infty} \int_{a}^{b} u(x y) d G(y) d F(x) \equiv E_{F}\left[E_{G} u(\tilde{x} \tilde{y})\right] .
$$

We define the derived utility function $v$ as the interior integral in equation $(1),{ }^{5}$

$$
v(x) \equiv \int_{a}^{b} u(x y) d G(y)=E u(x \tilde{y}) .
$$

\footnotetext{
${ }^{5}$ See Nachman (1982), who considers a more general relationship between $\tilde{x}$ and $\tilde{y}$. We specialize his measure to the case of multiplicative risks.
} 
Obviously $v$ is unique to the background risk $\tilde{y}$, and $v$ is increasing and concave since $u$ is. Thus, equation (1) can be written as $E u(\tilde{x} \tilde{y})=E v(\tilde{x})$. Decisions on $\tilde{x}$ made in the presence of the multiplicative risk $\tilde{y}$ under utility $u$ are isomorphic to decisions made on $\tilde{x}$ in isolation under the risk-averse utility $v^{6}$ Our goal is to determine when $v(x)$ is more risk averse than $u(x)$ :

$$
\frac{-v^{\prime \prime}(x)}{v^{\prime}(x)} \equiv \frac{-E\left[u^{\prime \prime}(x \tilde{y}) \tilde{y}^{2}\right]}{E\left[u^{\prime}(x \tilde{y}) \tilde{y}\right]} \geq \frac{-u^{\prime \prime}(x)}{u^{\prime}(x)} \quad \forall x .^{7}
$$

We let $r_{v}(x)$ and $r_{u}(x)$ denote the measures of absolute risk aversion for $v$ and $u$ respectively. Since we have a multiplicative background risk, it is often convenient to consider the corresponding measures of relative risk aversion, $R_{v}(x) \equiv x r_{v}(x)$ and $R_{u}(x) \equiv x r_{u}(x)$. Obviously, for any positive $x, r_{v}(x) \geq r_{u}(x)$ if and only if $R_{v}(x) \geq R_{u}(x)$.

Define preferences as being multiplicatively risk vulnerable, if an individual behaves more cautiously in the presence of any arbitrary independent multiplicative background risk such that $E \tilde{y} \leq 1 .^{8}$ That is, the individual is multiplicatively risk vulnerable if $R_{v}(x) \geq R_{u}(x) \quad \forall x$. We examine conditions for multiplicative risk vulnerability in detail in the following section.

For an arbitrary value of $x$, straightforward manipulation of the left-hand side of the inequality in (3) shows that

$$
R_{v}(x)=\int_{a}^{b} R_{u}(x y) d \eta_{x}(y), \text { where } \eta_{\mathrm{x}}(\mathrm{y}) \equiv \int_{0}^{\mathrm{y}} \frac{u^{\prime}(x t) t}{E\left[u^{\prime}(x \tilde{y}) \tilde{y}\right]} d G(t)
$$

\footnotetext{
${ }^{6}$ One needs to be careful with the terminology here. Although the derived utility $v$ is more risk averse than $u$, we should not say the individual is more risk averse in the presence of the background risk. The individual's risk aversion does not change. However, the individual behaves as if she had the more riskaverse utility $v$ with no background risk. This is why we prefer to label behavior as "more cautious."

${ }^{7}$ In order to keep the mathematics simple, we will take "more risk averse" to be in the weak sense of Pratt (1964). Stronger versions follow using well known (but tedious) methods.

${ }^{8}$ This definition perfectly mirrors the definition of risk vulnerability by Gollier and Pratt for the case of additive background risks.
} 
$\eta_{x}(y)$ is itself a risk-adjusted probability distribution. Defining $\hat{E}$ as the expectation operator based on the probability distribution $\eta_{x}(y)$, one obtains $R_{v}(x)=\hat{E}\left[R_{u}(x \tilde{y})\right]$. From equation (4), it follows trivially that $v$ inherits constant relative aversion (CRRA), whenever $u$ exhibits CRRA. It also turns out that $v$ inherits decreasing relative aversion from $u$, although the proof is less direct and we relegate it to an appendix.

More generally, equation (4) provides bounds for $R_{v}(x)$, such that $\inf \left\{R_{u}(x y)\right\} \leq R_{v}(x) \leq \sup \left\{R_{u}(x y)\right\}, \forall y \in \operatorname{Supp}(G)$. Thus, for example, if $R_{u}(x)>[<] 1$ $\forall x>0$, then $R_{v}(x)>[<] 1$ will hold for all $x$ as well. ${ }^{9}$

\section{Multiplicative Risk Vulnerability}

In this section, we derive necessary and sufficient conditions for multiplicative risk vulnerability. Since the conditions obtained are fairly complex, we also derive some sufficient conditions for which an undesirable independent multiplicative background risk leads to more cautious behavior.

Before proceeding to our Main Theorem, we require the following result, which is due to Gollier and Kimball (1996). This result also can be found in Gollier (2001).

\section{Diffidence Theorem (Gollier and Kimball): Let $\Lambda$ denote the set of all random variables} with support contained in the interval $[a, b]$ and let $f$ and $g$ be two real-valued functions. The following two conditions are equivalent:

(i) For any $\tilde{y} \in \Lambda, \quad E f(\tilde{y})=0 \Rightarrow \operatorname{Eg}(\tilde{y}) \geq 0$.

(ii) $\exists m \in \mathfrak{R}$ such that $g(y) \geq m f(y) \quad \forall y \in[a, b]$.

\footnotetext{
${ }^{9}$ Since many theoretical results in the literature depend on $R_{u}(x)>[<] 1$, this result shows that such results would also apply to the derived utility function $v$. For a discussion of these theoretical results, as well as an excellent survey on the empirical literature, see Meyer and Meyer (2005).
} 
We now can state the necessary and sufficient conditions for multiplicative risk vulnerability.

Main Theorem: Preferences are multiplicatively risk vulnerable if and only if for every $x>0$ and every $y \in[a, b]$, relative risk aversion is decreasing in wealth (DRRA) and

$$
u^{\prime}(x y) y\left[R_{u}(x y)-R_{u}(x)\right]-(y-1) u^{\prime}(x) x R_{u}{ }^{\prime}(x) \geq 0 .
$$

Proof: We need to examine properties on preferences $u$ such that

$$
R_{v}(x)=\frac{-E\left[u^{\prime \prime}(x \tilde{y}) x \tilde{y}^{2}\right]}{E\left[u^{\prime}(x \tilde{y}) \tilde{y}\right]} \geq R_{u}(x) \quad \forall x, \forall \tilde{y} \text { with } E \tilde{y} \leq 1 .
$$

Since $E \tilde{y} \leq 1$ allows for $\tilde{y}$ to be a constant $y<1$, the necessity of DRRA is apparent. Thus, it only remains to find conditions on $u$ such that (6) holds for $E \tilde{y}=1$ :

$$
E \tilde{y}=1 \Rightarrow-E\left[u "(x \tilde{y}) x \tilde{y}^{2}\right]-R_{u}(x) E\left[u^{\prime}(x \tilde{y}) \tilde{y}\right] \geq 0
$$

By the Diffidence Theorem, this statement is equivalent to finding a scalar $m$, such that

$$
-u^{\prime \prime}(x y) x y^{2}-R_{u}(x) u^{\prime}(x y) y \geq m(y-1) \quad \forall y,
$$

or equivalently,

$$
[\operatorname{sgn}(y-1)] u^{\prime}(x y) y\left[\frac{R_{u}(x y)-R_{u}(x)}{y-1}\right] \geq[\operatorname{sgn}(y-1)] m .
$$

Considering $y \rightarrow 1$, we see that the only candidate for $m$ is

$$
m=\left.\left[u^{\prime}(x y) y \frac{d R_{u}(x y)}{d y}\right]\right|_{y=1}=u^{\prime}(x) x R_{u}{ }^{\prime}(x) .
$$

Replacing $m$ in (8) above completes the proof. 
Remark: A close inspection of the proof shows that condition (5) alone, without DRRA, is necessary and sufficient for more cautious behavior if we restrict $E \tilde{y}=1{ }^{10}$ Also, under the restriction $E \tilde{y}=1$, it follows that $v$ will be less risk averse than $u$ if the inequality in (5) is reversed.

Obviously, condition (5) is not one that is easily verified. However, a few simple sufficient conditions for more cautious behavior follow easily, as we now show.

Corollary 1: Suppose that $R_{u}(x)$ is convex and that one of the following conditions holds $\forall(x, y) \in \operatorname{Supp}(F) \times \operatorname{Supp}(G)$ :

(i) $E \tilde{y} \leq 1, R_{u}(x y)>1$ and $R_{u}$ is decreasing,

or

$$
\text { (ii) } E \tilde{y}=1, R_{u}(x y)<1 \text { and } R_{u} \text { is increasing. }{ }^{11}
$$

Then $v$ is more risk averse than $u$.

Proof: Assume that $y>1$. The case where $y<1$ is similar. Under the assumptions in Corollary 1 , it follows that

$$
\left[\frac{R_{u}(x y)-R_{u}(x)}{x y-x}\right] \geq R_{u}{ }^{\prime}(x) \geq \frac{u^{\prime}(x)}{u^{\prime}(x y) y} R_{u}{ }^{\prime}(x) .
$$

The first inequality above follows from the convexity of relative risk aversion. To see the second inequality note first that

$$
\frac{\partial^{2} u(x y)}{\partial x \partial y}=\frac{\partial}{\partial y}\left[u^{\prime}(x y) y\right]=u^{\prime}(x y)\left[1-R_{u}(x y)\right] .
$$

\footnotetext{
${ }^{10}$ Likewise, the proof for the additive case in Gollier and Pratt (1996) shows that their inequality (14) $\left[u^{\prime}(x+\varepsilon)\left[r_{u}(x+\varepsilon)-r_{u}(x)\right]-\varepsilon u^{\prime}(x) r_{u}{ }^{\prime}(x) \geq 0 \forall x, \varepsilon\right]$, without DARA, is necessary and sufficient for more cautious behavior when $E \tilde{\varepsilon}=0$, as the authors point out. This inequality is the additive-background-risk equivalent of our inequality (5).

${ }^{11}$ It is not difficult to show that result also holds for $E \tilde{y}>1$. The same is true for Corollary 2 (ii).
} 
The second inequality in (11) follows from (12) in two particular cases: it follows if relative risk aversion is greater than one and decreasing, or if relative risk aversion is less than one and increasing. But (5) follows from (11). Hence, Corollary 1 holds.

From our remark following our Theorem, we can also derive conditions under which the derived utility $v$ is less risk averse than $u$. We present these as the following corollary, whose proof is similar to that of Corollary 1 and omitted here.

Corollary 2: Suppose that $R_{u}(x)$ is concave and that one of the following conditions holds $\forall(x, y) \in \operatorname{Supp}(F) \times \operatorname{Supp}(G)$ :

(i) $E \tilde{y} \leq 1, R_{u}(x y)>1$ and $R_{u}$ is increasing,

or (ii) $E \tilde{y}=1, R_{u}(x y)<1$ and $R_{u}$ is decreasing.

Then $v$ is less risk averse than $u$.

Of course, whether risk aversion exhibits constant-, increasing-, or decreasing relative risk aversion, or none of these, is an empirical question. Certainly constant relative risk aversion is very common in equilibrium asset-pricing models. But empirical support also exists for increasing relative risk aversion (e.g. Guiso and Paiella (2001)) as well as for decreasing relative risk aversion (e.g. Ogaki and Zhang (2001)). Whether relative risk aversion might be concave or convex in wealth has not received much attention at all until fairly recently. For example, Ait-Sahalia and Lo (2000) and Jackwerth (2000) examine market data and find evidence that $R_{u}$ is a (mostly) convex Ushaped function of wealth.

To illustrate Corollaries 1 and 2, consider the following examples:

Example 1: Suppose $E \tilde{y}=1$ and let $u$ be CARA, $u(x)=-e^{-k x}$ where $k>0$. Thus, $R_{u}{ }^{\prime}(x)=k$ and $R_{u}{ }^{\prime \prime}(x)=0$. Thus, $R_{u}$ is increasing and is both convex and concave. If 
we consider $\tilde{x}$ and $\tilde{y}$ such that $x y<1 / k \quad \forall(x, y) \in \operatorname{Supp}(F) \times \operatorname{Supp}(G)$, then $R_{u}(x y)<1$ and $v$ is more risk averse than $u$ by Corollary $1(i i)$. However, if $x y>1 / k$ $\forall(x, y) \in \operatorname{Supp}(F) \times \operatorname{Supp}(G)$, then $R_{u}(x y)>1$ and, hence, $v$ is less risk averse than $u$ by Corollary 2(i).

Example 2: Suppose $E \tilde{y}=1$ and let $u$ be quadratic, $u(x)=x-k x^{2}$ where $k>0$. We restrict $x<\frac{1}{2 k}$ so that marginal utility is positive. It is straightforward to show that $R_{u}(x)=2 k x(1-2 k x)^{-1}$ and that $R_{u}$ is both strictly increasing and convex. Moreover, $R_{u}(x y)<1$ if $x y<\frac{1}{4 k} \quad \forall(x, y) \in \operatorname{Supp}(F) \times \operatorname{Supp}(G)$, so that $v$ is more risk averse than $u$ by Corollary 1(ii). In other words, $v$ is more risk averse than $u$ over the first half of the relevant (upward-sloping) range of the quadratic utility function. On the other hand, if $\frac{1}{4 k}<x y<\frac{1}{2 k} \forall(x, y) \in \operatorname{Supp}(F) \times \operatorname{Supp}(G)$, then $R_{u}(x y)>1$, but we cannot apply Corollary 1 (since $R_{u}$ is increasing) or Corollary 2 (since $R_{u}$ is convex).

Both utility functions above belong to the so-called HARA class of utility, as does CRRA utility. To further explore the HARA class, let $u(x)=\xi\left(\eta+\frac{x}{\gamma}\right)^{1-\gamma}$, where $\eta+\frac{x}{\gamma}>0$ and $\frac{\xi(1-\gamma)}{\gamma}>0$. Straightforward calculations show that $R_{u}{ }^{\prime}(x)=\eta\left(\eta+\frac{x}{\gamma}\right)^{-2}$ and that $R_{u}{ }^{\prime \prime}(x)=-\left[\frac{2}{\gamma}\left(\eta+\frac{x}{\gamma}\right)^{-1}\right] R_{u}{ }^{\prime}(x)$. Thus, for the case of constant absolute risk aversion $(\gamma \rightarrow \infty)$, we obtain $R_{u}{ }^{\prime}(x)=k$ and $R_{u}{ }^{\prime \prime}(x)=0$, as in Example 1. If we have increasing absolute risk aversion, then we must have $\gamma<0$ and $\eta>0$. It follows that $R_{u}{ }^{\prime}(x)>0$ and $R_{u}{ }^{\prime \prime}(x)>0$, so that we must have $R_{u}$ increasing and convex, as is the case with our quadratic utility in Example 2. On the other hand, if we have decreasing absolute risk aversion (DARA), then $\gamma>0$. Hence, $\operatorname{sgn} R_{u}{ }^{\prime \prime}(x)=-\operatorname{sgn} R_{u}{ }^{\prime}(x)$. Consequently, we must have $R_{u}$ either (i) constant, (ii) decreasing and convex, or (iii) increasing and concave. Since HARA is such a common form for preferences, we summarize these results in the following Corollary, corresponding to cases (i) - (iii) above: 
Corollary 3: Suppose that u belongs to the HARA class of utility and that absolute risk aversion is decreasing.

(i) If u satisfies CRRA, then $v$ and $u$ are equivalent.

(ii) Let E $\tilde{y} \leq 1$. If $R_{u}>1$ and decreasing, then $v$ is more risk averse than $u$.

(iii) Let E $\tilde{y} \leq 1$. If $R_{u}>1$ and increasing, then $v$ is less risk averse than $u$.

Note that we have $R_{u}>1$ in our example in the introduction of this paper (see Table 1). The conditions (i), (ii) and (iii) above correspond to the three cases considered in our example, with $a=0, a=-25$ and $a=+25$ respectively.

\section{The Affiliated Utility Function}

In this section, we use the relationship that $\ln (x y)=\ln x+\ln y$, which allows us to adapt several results from the case of additive background risks. In order to accomplish this, we introduce the affiliated utility function, $\hat{u}$, which we define such that $u(x)=\hat{u}(\ln x)$, for all $x>0$. Equivalently, we can substitute $\theta=\ln x$ to write $\hat{u}(\theta) \equiv u\left(e^{\theta}\right) \quad \forall \theta \in \mathfrak{R}$. Since $u(x y)=\hat{u}(\ln x+\ln y)$, we can examine properties for the additive risks $\ln \tilde{x}+\ln \tilde{y}$.

Let $\hat{r}(\theta)$ denote the absolute risk aversion for $\hat{u}(\theta)$, i.e. $\hat{r}(\theta)=-\hat{u}^{\prime \prime}(\theta) / \hat{u}^{\prime}(\theta)$. Straightforward calculations show that

$$
R_{u}(x)=1-\frac{\hat{u}^{\prime \prime}(\ln x)}{\hat{u}^{\prime}(\ln x)}=1+\hat{r}_{u}(\ln x) .
$$

Note that $R_{u}(x)<1$ implies that $\hat{r}_{u}(\ln x)<0$. By examining the nature of $\hat{r}$, we will be able to adapt several existing results on additive background risk to the multiplicative case. 
Define $\hat{v}(\ln x) \equiv E \hat{u}(\ln x+\ln \tilde{y}) . \quad$ It follows that $\hat{v}(\ln x)=v(x) . \quad$ In a manner analogous to equation (13) we can derive

$$
R_{v}(x)=1-\frac{E \hat{u}^{\prime \prime}(\ln x+\ln \tilde{y})}{E \hat{u}^{\prime}(\ln x+\ln \tilde{y})} \equiv 1+\hat{r}_{v}(\ln x) .
$$

From (13) and (14), we see that $R_{v}(x) \geq R_{u}(x)$ if and only if $\hat{r}_{v}(\ln x) \geq \hat{r}_{u}(\ln x)$. For the case where $R_{u}>1$, this implies that $\hat{u}$ is (additively) risk vulnerable if and only $u$ is multiplicatively risk vulnerable. For the case where $R_{u}<1$, so that $\hat{u}$ is risk loving, we still can interpret $\hat{r}_{v}>\hat{r}_{u}$ as meaning " $\hat{v}$ is more risk averse than $\hat{u}$," but in the sense of being less risk loving. From (13) and (14) it also follows that $R_{t}(x)$ is decreasing in $x$ if and only if $\hat{r}_{t}(\ln x)$ is decreasing in $\ln x, t=u, v$.

If we wish to extend results from the literature on additive background risks to the case of multiplicative ones, we need to relate our setting to that of Gollier and Pratt (1996). In addition to not requiring risk aversion of $\hat{u}$, we restrict ourselves to additive background risks $\ln \tilde{y}$ for which $E \tilde{y} \leq 1$, implying $E \ln \tilde{y}<0$, whereas Gollier and Pratt consider background risks such that $E \ln \tilde{y} \leq 0$, using our notation.

Perhaps surprisingly, Gollier's and Pratt's proof of their necessary and sufficient conditions for risk vulnerability does not require risk aversion. It requires only that utility be strictly increasing. This might not seem as surprising if we note that our proof of the Main Theorem also does not require risk aversion. Also, since $E \ln \tilde{y}<0$ allows for $E \ln \tilde{y}$ to be arbitrarily close to zero, it follows that the conditions for additive risk vulnerability of $\hat{u}$ in the sense of Gollier and Pratt (which would require $E \ln \tilde{y} \leq 0$ ) are no different than the conditions for more cautious behavior when $E \ln \tilde{y}<0 .{ }^{12}$

Since risk vulnerability also is not an easy trait to verify in the additivebackground-risk case, Gollier and Pratt provide two sufficient conditions that lead to

\footnotetext{
12 Applying Gollier and Pratt (1996, inequality (14)) to $\hat{u}(\ln x+\ln y)$ yields necessary and sufficient conditions for $\hat{u}$ to be (additively) risk vulnerable, which in turn will imply that $u$ is multiplicatively risk vulnerable. Note however that $u$ itself need not be additively risk vulnerable.
} 
more cautious behavior. Unfortunately for the applicability of the affiliated utility function, these simple sufficient conditions do require that $\hat{u}$ be risk averse. Thus, we can only apply these sufficient conditions when $R_{u}>1$.

This leads to the following two sufficient conditions on the affiliated utility function $\hat{u}$ to ensure that preferences $u$ are multiplicatively risk vulnerable. They follow directly from Corollary 1 and section 5.4 in Gollier and Pratt (1996).

\section{Proposition 1: Let $R_{u}(x)>1 \forall x$. Then u is multiplicatively risk vulnerable if either}

(i) $\hat{r}_{u}$ is decreasing and convex,

or (ii) $\hat{u}$ exhibits standard risk aversion (see Kimball, 1993, and below).

In some instances, we might be able to check the conditions on the affiliated utility function $\hat{u}$ in Proposition 1 directly. However, we are somewhat limited even in this case, since known sufficient conditions for additive background risk only apply when we have $\hat{u}$ risk averse $\left(R_{u}>1\right)$ and they only apply for $v$ more risk averse than $u$. As we saw from the examples of the previous section, several circumstances with $R_{u}>1$ also led to $v$ being less risk averse than $u$. Thus, we are unsure of the current usefulness of the affiliated utility. However, as new results on additive risks become available, this might change.

As an example of how we can use affiliated utility, consider the case of CARA, $u(x)=-e^{-k x}, k>0$. It follows in this case that $\hat{u}(\theta)=-\exp \left(-k e^{\theta}\right)$. Straightforward calculations show that $\hat{u}$ is concave if and only if $e^{\theta}=x>\frac{1}{k}$, which as we saw previously equates to $R_{u}>1$. However, $\hat{r}_{u}(\theta)=k e^{\theta}-1$, which is increasing so that Proposition 1 will not apply.

For the more general case of HARA utility, with $u(x)=\xi\left(\eta+\frac{x}{\gamma}\right)^{1-\gamma}$, we have $\hat{u}(\theta)=\xi\left(\eta+\frac{1}{\gamma} e^{\theta}\right)^{1-\gamma}$. Straightforward calculations show that $\hat{r}(\theta)=e^{\theta}\left(\eta+\frac{1}{\gamma} e^{\theta}\right)^{-1}-1$, which is easily seen to be positive if and only if $R_{u}>1$. Further calculation shows that 
$\hat{r}^{\prime}(\theta)<0$ if and only if we have $\gamma>0$ and $\eta<0$, and that in this case $\hat{r}^{\prime \prime}(\theta)>0$. But $\gamma>0$ and $\eta<0$ imply respectively DARA and DRRA for $u$. Thus, DARA and DRRA within the HARA class of preferences, with $R_{u}>1$, implies that $\hat{r}(\theta)$ is decreasing and convex, and hence $v$ is more risk averse than $u$ by Proposition 1(i). Of course, we already showed this as Corollary 3(ii).

Typically, we find it easier to deal with properties of $u$ directly, rather than properties of $\hat{u}$. Still, this tool might be useful for seemingly odd utility functions that have a well-understood form for their affiliated utility. For example, suppose that $u(x) \equiv \ln (\ln x)$ for $x>1$. Then $\hat{u}(\theta)=\ln \theta$. Or suppose that $u(x) \equiv-(a+b \ln x)^{-1}$, where $a$ and $b$ are positive constants and $x>0$. Then $\hat{u}(\theta) \equiv-(a+b \theta)^{-1}$. In both cases $u$ is multiplicatively risk vulnerable as its affiliated utility $\hat{u}$ satisfies both parts $(i)$ and (ii) of Proposition 1.

Although Proposition 1 does yield a useful result, it is not clear that much more can be gleaned from the affiliated utility function. It is limited by the extant results in the literature on additive background risks, and this poses two problems. First, except for the necessary and sufficient conditions for risk vulnerability, all of the other results that we are aware of require risk aversion, so that they only can be adapted to the multiplicativebackground-risk case when relative risk aversion exceeds one. Second, as we saw in Corollary 2, seemingly reasonable assumptions about preferences can lead to lesscautious behavior in the presence of a multiplicative background risk. In such cases, the derived affiliated utility function $\hat{v}$ will necessarily be less risk averse than $\hat{u}$. There is no literature that we are aware of that provides sufficient conditions for less-cautious behavior in the presence of an additive background risk. ${ }^{13}$ As more results become available for the additive case, it may be that many of these can be applied to the

\footnotetext{
13 The only exception is the necessary and sufficient condition of Gollier and Pratt (1996) for risk vulnerability when restricted to the case where $E \tilde{\varepsilon}=0$, which is achieved by simply reversing their inequality in their expression (14). But this case is of no interest here, since under the assumption that $E \tilde{y} \leq 1$, we never have $E \ln \tilde{y}=0$.
} 
multiplicative case via use of affiliated utility. We thus feel it is a potentially useful tool, but limited by the current literature.

\section{Concluding Remarks}

The notion that markets are complete is a mathematical nicety that does not hold true in practice. Many types of political, human-capital and social risks, as well as some financial risks, are not tradeable. Obviously, many of these risks might be hedged indirectly via so-called "cross hedging." However, even when such background risks are independent of other risks and cannot be hedged, they still may have an impact upon risktaking strategies that are within the control of the economic agent. Much has been done over the past twenty years in examining the effects of additive background risks, but not much has been done to systematically study economic decision making in the presence of a multiplicative background risk.

As the examples in our introduction illustrate, models with such multiplicative background risks are not hard to find. Whereas properties of absolute risk aversion play a key role in analyzing the effects of an additive background risk, properties of relative risk aversion are important in examining behavior in the presence of a multiplicative background risk.

As shown in the paper, results for the case of a multiplicative background risk do not simply "mirror" those for the case where the background risk is additive. Indeed, there are no empirical studies that even suggest that multiplicative risk vulnerability is some type of canonical behavior, which stands in contrast to the additive case. Moreover, whereas sufficient conditions for risk vulnerability in the additive case are satisfied by most of the commonly used forms of utility, the same is not true in the multiplicative case. To be sure, the sufficient conditions for multiplicative risk vulnerability are common assumed; but so are the conditions guaranteeing that the 
individual will behave in a less-cautious manner in the presence of a multiplicative background risk.

We also introduced the affiliated utility function, which converts the multiplicative risk $\tilde{x} \tilde{y}$ into and additive one, $\ln \tilde{x}+\ln \tilde{y}$. Although this device was seen to be somewhat limited, given the current literature, it does provide us with a few new results plus a new perspective on the multiplicative background risk case vis-à-vis the case of additive background risk. As we learn more about the inherent properties of preferences in the additive case, the affiliated utility may become an invaluable tool.

This paper is just one step towards developing a more comprehensive theory of background risk and its implications. Of course, we made the simplifying assumption that the background risk was independent. More realistically, background risks are likely to be correlated with other risks in an individual's wealth portfolio. Plus, we limited the analysis here to consideration of multiplicative background risks. More general formulations, as well as possibilities for two or more background risks to be present simultaneously, are also reasonable extensions of the theory. However, these topics are beyond the scope of this paper and left to future research. 


\section{Appendix}

Claim: $R_{v}(x)$ is decreasing (i.e. DRRA) whenever $R_{u}(x)$ is decreasing.

Proof: Note that DRRA holds if and only if $P_{u}(x) \geq 1+R_{u}(x)$. Thus we must show that:

$$
P_{u}(x) \geq 1+R_{u}(x) \Rightarrow P_{v}(x) \geq 1+R_{v}(x) \quad \forall x,
$$

Or equivalently

$$
\frac{-E u^{\prime \prime \prime}(x \tilde{y}) \tilde{y}^{3} x}{E u u^{\prime \prime}(x \tilde{y}) \tilde{y}^{2}} \geq \frac{-E u^{\prime \prime}(x \tilde{y}) \tilde{y}^{2} x}{E u^{\prime}(x \tilde{y}) \tilde{y}}+1 .
$$

Inequality (A2) is equivalent to the following:

$$
E\left[u^{\prime \prime}(x \tilde{y}) \tilde{y}^{2} x+(\lambda-1) u^{\prime}(x \tilde{y}) \tilde{y}\right]=0 \Rightarrow E\left[u{ }^{\prime \prime}(x \tilde{y}) \tilde{y}^{3} x+\lambda u u^{\prime \prime}(x \tilde{y}) \tilde{y}^{2}\right] \geq 0 .
$$

By the Diffidence Theorem, (see Section 3 in the text), (A3) will hold if we can find a real number $m$, with

$$
u^{\prime \prime \prime}(x y) y^{3} x+\lambda u^{\prime \prime}(x y) y^{2} \geq m\left[u u^{\prime \prime}(x y) y^{2} x+(\lambda-1) u^{\prime}(x y) y\right] \quad \forall y \in[a, b] .
$$

The left-hand side of (A4) can be written as

$$
\frac{x y u^{\prime \prime}(x y)}{u^{\prime}(x y)} \quad \frac{u^{\prime}(x y) y}{x}\left[\frac{x y u^{\prime \prime}(x y)}{u^{\prime \prime}(x y)}+\lambda\right]=-R_{u}(x y) \frac{u^{\prime}(x y) y}{x}\left[\lambda-P_{u}(x y)\right] .
$$

Since $P_{u}(x) \geq 1+R_{u}(x)$, it follows from (A4) and (A5) that

$$
u^{\prime \prime \prime}(x y) y^{3} x+\lambda u u^{\prime \prime}(x y) y^{2} \geq-R_{u}(x y) \frac{u^{\prime}(x y) y}{x}\left[\lambda-1-R_{u}(x y)\right] .
$$

From (A4) and (A6), we would be done if we could find an $m$, such that

$$
\begin{array}{r}
-R_{u}(x y) \frac{u^{\prime}(x y) y}{x}\left[\lambda-1-R_{u}(x y)\right] \geq m\left[u{ }^{\prime \prime}(x y) y^{2} x+(\lambda-1) u^{\prime}(x y) y\right] \\
=m u^{\prime}(x y) y\left[\lambda-1-R_{u}(x y)\right] .
\end{array}
$$

This follows by taking $m=(1-\lambda) / x$, since we then obtain (31) is equivalent to

$$
-R_{u}(x y)\left[\lambda-1-R_{u}(x y)\right]+(\lambda-1)\left[\lambda-1-R_{u}(x y)\right]=\left[\lambda-1-R_{u}(x y)\right]^{2} \geq 0 .
$$

Hence, (A1) holds and $v$ exhibits decreasing relative risk aversion. 


\section{References}

Aït-Sahalia, Y. and A.W. Lo, (2000), "Nonparametric Risk Management and Implied Risk Aversion," Journal of Econometrics 94, 9-51.

Arrow, K.J., (1971), Essays in the Theory of Risk Bearing, Chicago: Markum Publishing Company.

Doherty, N. A. and H. Schlesinger, (1983), "Optimal Insurance in Incomplete Markets," Journal of Political Economy, 91, 1045-1054.

Eeckhoudt, L. and M.S. Kimball, (1992), "Background Risk, Prudence and the Demand for Insurance," in: Dionne, G. (Ed.), Contributions to Insurance Economics, Boston: Kluwer Academic Publishers, pp. 239-254.

Finkelshtain, I., O. Kella and M. Scarsini, (1999), "On Risk Aversion with Two Risks," Journal of Mathematical Economics 31, 239-250.

Gollier, C., (2001), The Economics of Risk and Time, Cambridge: MIT Press.

Gollier, C. and M. Kimball (1996), "Towards a Systematic Approach to the Economics of Uncertainty: Characterizing Utility Functions," unpublished working paper, University of Michigan.

Gollier, C. and J. W. Pratt, (1996), "Risk Vulnerability and the Tempering Effect of Background Risk," Econometrica, 64, 1109-1124.

Guiso, L., T. Japelli and D. Terlizzese, (1996), "Income Risk, Borrowing Constraints and Portfolio Choice," American Economic Review 86, 158-172.

Guiso, L. and M. Paiella, (2001), "Risk Aversion, Wealth and Financial Market Imperfections," CEPR Discussion Paper No. 2728.

Jackwerth, J.C., (2000), "Recovering Risk Aversion from Option Prices and Realized Returns," Review of Financial Studies 13, 433-451.

Kihlstrom, R., D. Romer and S. Williams, (1981), "Risk Aversion with Random Initial Wealth," Econometrica, 49, 911-920.

Kimball, M.S., (1990), "Precautionary Savings in the Small and in the Large." Econometrica 58, 53-73.

Kimball, M.S., (1993), “Standard Risk Aversion,” Econometrica, 61, 589-611. 
Meyer, D.J. and J. Meyer (2005), "Relative Risk Aversion: What Do We Know?" Journal of Risk and Uncertainty, forthcoming

Nachman, D., (1982), “Preservation of 'More Risk Averse' under Expectations," Journal of Economic Theory, 28, 361-368.

Ogaki, M. and Q. Zhiang, (2001), "Decreasing Relative Risk Aversion and Tests of Risk Sharing," Econometrica 69, 515-526.

Pratt, J.W., (1964), "Risk Aversion in the Small and in the Large," Econometrica 32, 122-136.

Pratt, J.W., (1988), “Aversion to One Risk in the Presence of Others," Journal of Risk and Uncertainty 1, 395-413.

Pratt, J. W. and R. Zeckhauser, (1987), “Proper Risk Aversion,” Econometrica, 55, 143154.

Ross, S. A., (1981), "Some Stronger Measures of Risk Aversion in the Small and in the Large with Applications," Econometrica 49, 621-638.

Rothschild, M., and J. Stiglitz, (1970), “Increasing Risk: I. A Definition,” Journal of Economic Theory 2, 225-243. 